\section{Research Square}

Preprints are preliminary reports that have not undergone peer review.

They should not be considered conclusive, used to inform clinical practice, or referenced by the media as validated information.

\title{
Clinical Efficacy and Diagnostic Value of Metagenomic Next- Generation Sequencing for Rapid Pathogen Detection in Patients with Suspected Infectious Diseases
}

\author{
Yang-hua Xiao \\ The First Affiliated Hospital of Nanchang University \\ Mei-fang Liu \\ The First Affiliated Hospital of Nanchang University \\ Hong-wen Wu \\ The First Affiliated Hospital of Nanchang University \\ De-rong Xu \\ The First Affiliated Hospital of Nanchang University \\ Wei Zhang \\ The First Affiliated Hospital of Nanchang University \\ Rui Zhao ( $\nabla$ zhaoruisc@163.com ) \\ The First Affiliated Hospital of Nanchang University
}

\section{Research Article}

Keywords: Metagenomic next-generation sequencing, infection, validation, diagnostic, outcome

Posted Date: March 8th, 2022

DOI: https://doi.org/10.21203/rs.3.rs-1314343/v1

License: @) (1) This work is licensed under a Creative Commons Attribution 4.0 International License. Read Full License 


\section{Abstract \\ Background}

Metagenomic next-generation sequencing (mNGS) is a powerful method for the diagnosis of suspected infections, avoiding empirical broad-spectrum therapy and lessening the risk of drug resistance. However, little is known about its clinical efficacy and diagnostic value when used in routine practice.

\section{Methods}

A reference panel of 10 microorganisms was designed to characterize mNGS test accuracy. Retrospective study was performed to compare the diagnostic value of mNGS tests and culture in infectious disease, and evaluated the clinical efficacy of mNGS based on interpretation of mNGS results by clinicians as well as whether leading to a favorable clinical outcome.

\section{Results}

At a typical sequencing depth and the concentration of human DNA up to $100,000 \mathrm{cell} / \mathrm{ml}$, the sequencing platform can still detect pathogens at concentration of $400 \mathrm{CFU} / \mathrm{ml}$. Among 518 patients, the sensitivity and specificity of mNGS for diagnosing infectious disease were $79.49 \%$ and $82.20 \%$, respectively. The bacteria and fungus detection rate (apart from candida) were significantly higher than culture $(P<0.01)$. Test sensitivity and specificity of mNGS were 78.40 and $95.27 \%$ for bacteria and 75.23 and $93.95 \%$ for fungi, respectively. mNGS was especially practical in identification of atypical pathogens, contributing a positive impact in $267(51.54 \%)$ cases in which 142 cases where mNGS results directly led to a change in treatment.

\section{Conclusions}

mNGS significantly improved sensitivity for bacteria and fungi identification, is a valuable tool for for identifying infectious patients and could optimize antimicrobial therapy to improve the clinical outcomes. Further multicenter and prospective studies with larger samples are needed to validate the clinical efficacy and diagnostic value of mNGS.

\section{Background}

Infectious diseases remain a leading cause of morbidity and mortality to human race(1), and present a rising challenge to public security. The most threatening infections such as pneumonia, sepsis and endocarditis occur when diffusion of the microorganism into the bloodstream occurs. Each hour of delay in the use of antibiotic has been associated with increased mortality(2). The miserable prognosis of infectious diseases highlights the continuing challenges of early accurate diagnosis and targeted antibiotic therapy of patients with infectious diseases. Conventional culture is considered the gold standard for etiological diagnosis. But viruses are unculturable and some fastidious microorganisms are time-consuming to culture (for example, mycobacteria, treponema pallidum, and talaromyces marneffei) $(3,4)$. The sensitivity of culture depends largely on the disease stage and whether or not patients have been prior exposed to antibiotics.

Rapid detection of unknown pathogenic microorganisms and antibiotic resistance identification in patients with suspected infectious diseases is critical to the etiological diagnosis and guide correctly clinical treatment. Multiplex PCR is an alternative diagnostic method to culture, which have been studied applied on infectious diseases in body fluid samples including blood and bronchoalveolar lavage $(\mathrm{BAL})(5,6)$. But prior selecting known target pathogens greatly limits the application of multiplex PCR in detecting unknown pathogens and mixed infections. Unexpected, rare, or novel pathogens can evade detection by multiplex PCR. Conventional microbiologic detection methods are difficult to reveal the etiologies of complicated infectious diseases, which present with indistinguishable clinical symptoms(7). These limitations may delay antimicrobial treatment, prolong hospitalizations and readmissions, and even lead to erroneous drug overuse, increase resistance to multiple antibiotics and healthcare costs, complicate treatment. These are currently insurmountable obstacles to the diagnosis and treatment of infectious diseases. Aggressive antimicrobial adjustment based on timely and accurately microbiology results is necessary.

Metagenomic next-generation sequencing (mNGS) is a hypothesis-free and untargeted molecular approach that can theoretically identify all pathogen nucleic acid and simultaneously predict antibiotic resistance gene targets in different body fluids. As a promising tool, mNGS has greatly expanded the scope of infectious disease pathogen investigation, which enables detect 1,250 clinical pathogens(virus, bacteria, fungi and/or parasites)(8), especially suitable for atypical and complexed etiologies of infectious diseases. However, the sensitivity of mNGS is heavily depend on the level of human host background and the efficiency of extracting nucleic acids from different samples. Interpretation of mNGS results demands further validation by a large number of clinical trials. Present study relevant to clinical application and impact has mostly limited to case reports or small-scale cohort studies(9-15), most of which have focused on pathogen detection from single type of sample. To bridge this research gap, we performed a multi-type retrospective study to assess the clinical efficacy and value of mNGS test for rapid pathogen detection in patients with suspected infectious diseases, explore its application in clinical microbiology and considerations for implementation of mNGS in a clinical laboratory.

In this study, we performed an accuracy validation experiment on our platform's capacity to analysis and interpretation of sequencing data. The clinical diagnostic performance of mNGS was compared to results from conventional microbiological testing, and verified by comparing the composite standard. The clinical impact of mNGS was determined based on the decision of the treatment team and whether leading to a favorable clinical outcome. 


\section{Methods}

\section{Analytical validation strategy}

A reference panel of 10 microorganisms (Listeria grayif, Haemophilus parainfluenzae, Acinetobacter junii, Rhodococcus equi, Micrococcus luteus, Neisseria sicca, Pseudomonas fluorescens, Aeromonoas hydrophila, Legionella pneumophila, Candida lusitaniae) was designed to characterize test accuracy. Six reference samples were used to validate accuracy. D0 was a negative blank control. The concentration of human DNA in plasma can exceed 1,000-fold, which can affect the sensitivity of sequencing-based assays(16). Therefore, five reference samples (D1-D5) containing different concentrations of pathogen DNA and human DNA. The concentrations of pathogens range from 400,000 to $400 \mathrm{CFU} / \mathrm{ml}$ and had human DNA that ranged from 1000 to 100,000 cells/ml. It also included both frequent colonizers and background microorganisms that are commonly found as high-level environmental contaminants. Test accuracy was characterized in three different sample matrices. D1, D2 and D3 representing 'high human' DNA samples, containing 100,000 cells/ml human DNA. The concentration of human DNA of D4 was 10,000 cells/ml, which representing 'medium human' DNA sample. The concentration of human DNA of D5 was 1000 cells/ml, which representing 'low human' DNA sample. In order to determine the read of pathogens in a sample without bias, the human DNA and pathogen DNA were randomly distributed into the partitions. The details of reference panel were presented in Supplementary Table S1. All reference samples require nucleic acid extraction as with body fluid samples.

\section{Study Population}

This study was ethically approved by the institutional review board of the First Affiliated Hospital of Nanchang University. All methods were performed in accordance with the relevant guidelines and regulations. We performed a retrospective cohort study of 518 cases suspected of acute or chronic infection at the First Affiliated Hospital of Nanchang University (Jiangxi Province, China) between December 2020 and October 2021. For all cases, there was either high suspected infectious clinical manifestations or infectious etiology. Cases were excluded if patient confirmed noninfectious disease before testing. Samples were excluded if sample failing to pass quality control of mNGS. Flowchart of cases inclusion and exclusion was shown in Fig. $1 \mathrm{a}$. 0 f 518 cases, 407 was performed microbiological culture in parallel. The same eligible sample divided or sampling interval less than 48 hours, which was defined as having paired mNGS and conventional microbiological culture results in our study. In addition to conventional microbiological culture, additional pathogen detection methods were only preformed for patients with highly suspected infections (for example, nucleic acid detection of pathogenic microorganism, quantitativePCR, enzyme-linked immunospot assay, GeneXpert TB Test, and galactomannan test, T-spot, imaging, orthogonal microbial testing of other sample types collected contemporaneously from the same patient). Final clinical diagnosis was made by clinicians based on all microbiological testing and a longitudinal review of the patient's clinical characteristics. Consider the possibility of repeated mNGS testing in the same patient for different period or reasons including patient monitoring or suspected false-negative results from the first mNGS test, the clinical efficacy and diagnostic value were mainly evaluated based on the first mNGS test per patient. The process of data collection was non-selective and continuous.

\section{Sample processing and Nucleic Acid Extraction}

The collected bronchoalveolar lavage samples were sent to the clinical microbiology lab and histopathology lab within $1 \mathrm{~h}$ after the collection. Volumes of $0.3 \mathrm{ml}$ of cerebrospinal fluid were drawn from patient, placed in a $1.5 \mathrm{ml}$ centrifuge tube, centrifuged at $5000 \mathrm{~g}$ for 10 minutes at $4^{\circ} \mathrm{C}$. All other body fluid samples of $0.3 \mathrm{ml}$ were placed in a $1.5 \mathrm{ml}$ centrifuge tube, centrifuged at $1600 \mathrm{~g}$ for 10 minutes at $4^{\circ} \mathrm{C}$, then draw $0.25 \mathrm{ml}$ supernatant into another $1.5 \mathrm{ml}$ centrifuge tube, centrifuged at $16000 \mathrm{~g}$ for 5 minutes at $4^{\circ} \mathrm{C}$. All samples were transferred to new sterile 1.5 -mL centrifuge tubes and DNA was extracted using the HUGO DNA and RNA Kit (YG-002, Hugo Biotech, Beijing, Beijing, CHINA), following the manufacturer's operational manual. The extracted nucleic acid samples were used for the construction of DNA libraries.

\section{Library construction}

DNA libraries were constructed through end-repair, adapter ligation, PCR amplification and purification. Library quality inspection including the determination of Library concentration and Library fragment size. An Agilent 2100 Bioanalyzer (Agilent Technologies, Santa Clara, CA, USA) and A Qubit 4 Fluorometer (Thermo Fisher Scientific, Singapore) were used for quality control of the DNA libraries length (200-500bp) and the library concentration (no less than $1 \mathrm{ng} / \mu \mathrm{l})$, respectively. Libraries were prepared and then sequenced using the NextSeqTM 550Dx (Illumina Technologies, San Diego, CA, USA).

\section{Criteria for Positive mNGS Result}

Excluding the normal flora of oral cavity, respiratory tract or the skin. For common contaminating organisms such as Prevotella pallens, Treponema maltophilum, Dialister invisus, Neisseria subflava, Rothia dentocariosa, etc. was considered negative in this study. The number of sequences standardized stringently mapped read number at the species level (SDSMRN) of microorganisms in sample is higher than in the negative control group. For different types of microorganisms, the positive criteria were set as follows: Bacterial (mycobacterium excluded), fungus, virus, mycoplasma, chlamydia, parasite: mNGS identified a microbe whose SDSMRN 3. Mycobacterium was considered positive when at least 1 SDSMRN due to the difficulty of nucleic acid extraction and low likelihood for contamination. The detailed sequencing data after processing is described in Supplemental Table S2.

\section{Statistical Analysis}

Comparative analysis was conducted by Pearson $\chi 2$ test. Discrete variables were analyzed with Fisher's exact test, where appropriate. The $2 \times 2$ contingency tables were established to determine the sensitivity and specificity of mNGS and culture for different pathogens. The results are presented with $95 \%$ confidence intervals (Cls). Data analyses were conducted using SPSS 25.0 software (SPSS Inc, Chicago, IL, USA), a two-tailed value of $P<0.05$ was considered significant.

\section{Results}




\section{Accuracy Validation}

The sensitivity of sequencing-based tests depends on the number of sequencing reads obtained. In validation tests, all different concentrations of pathogens were detected. The number of sequencing reads were determined for each of the ten reference microorganisms in each of the high-humanDNA(D1-D3), medium-human-DNA(D4) and low-human-DNA(D5) plasma matrices. At a typical sequencing depth and the concentration of human DNA up to 100,000 cells $/ \mathrm{ml}$, our sequencing platform can still detect pathogens at concentration of $400 \mathrm{CFU} / \mathrm{ml}$. When the concentration of human DNA was 10000 or 1000 cells $/ \mathrm{ml}$ and the concentration of pathogen remained $1000 \mathrm{CFU} / \mathrm{ml}$, the number of readings increased significantly (D4 and D5). This suggests that human DNA concentration indeed can affect the sensitivity of sequencing. The results of accuracy validation were presented in Table 1.

\section{Sample and Patient Characteristics}

The demographic features and characteristic baselines in the current study were presented in Table 2 . Within the 518 patients included, 341 were males and 177 were females. 139 patients $(26.83 \%)$ were admitted into the intensive care unit. All patients had infection-related symptoms, with the most common symptoms being fever (47.30\%), cough (20.66\%), and increased sputum production (14.29\%) (Table 2). Among them, 46.91\% (243/518) patients had underlying diseases, including hypertension (125 cases), diabetes (53 cases), chronic liver diseases (51 cases), chronic lung diseases (30 cases), cerebrovascular diseases (24 cases), cardiovascular diseases (13 cases), chronic kidney diseases (13 cases), organ transplantation (6 cases), autoimmunity diseases ( 5 cases). A total of $94.59 \%$ (490/518) patients with final diagnosis of infection and $5.41 \%(28 / 518)$ patients were diagnosed as non-infectious diseases. Most of our sample types are blood, with 200 of 518 (38.61\%) from blood, 154 of 518 (29.73\%) from bronchoalveolar, 62 of 518 (11.97\%) from cerebrospinal, 29 of 518 (5.60\%) from sputum, 29 of 518 (5.60\%) from peritoneal, 17 of 518 (3.86\%) from bone marrow, 7 of 566 (1.35\%) from pleural and 20 of 566 (3.28\%) from other body fluids (Fig. 1b). Of 518 samples, 16.79\% (87 of 518) had no microorganisms reported, a single species was detected in most cases (Fig. 1c). More than two pathogens were detected in samples of 266 patients (51.35\%) using mNGS. Based on Illumina data, the ratio of microbial reads in sputum and bronchoalveolar lavage fluid was higher than that in other types of samples, indicating that sputum and bronchoalveolar lavage fluid had a higher pathogen load (Fig. 1d).

\section{Comparison of Pathogenic Detection Between mNGS and culture}

From sample collected to result analysis, the time consumption of mNGS test was shorter than that of the culture (Fig. 1e). In order to compare the diagnostic efficiency of mNGS and culture, 407 samples with paired culture test were included for further study. In our results, mNGS and conventional microbiological culture were both positive in 78 of 407 (19.16\%) cases and were both negative in 75 of 407 (18.43\%) cases (Fig. 2a). Two hundred and fiftythree samples were positive by mNGS only $(62.16 \%)$ and 1 was positive by culture only $(0.25 \%)$. For double-positive samples, the 8 results were completely matched and 9 results were totally mismatched. The remaining 61 cases were found to be partly matched which means at least 1 overlap of pathogens when multi-microbial results were observed. Among pathogens detected by mNGS and culture, Klebsiella pneumoniae $(n=60)$ and Candida $(n=65)$ were the most frequently detected bacteria and fungus, respectively (Fig. 2b). The bacteria and fungus, apart from candida, demonstrated a superior positivity rate in mNGS than that in culture $(P<0.01)$.

\section{Diagnostic Performance Comparison of mNGS and Culture}

A composite standard was applied in the evaluation of diagnostic Performance that incorporated additional results from (1) microbial culture and orthogonal microbial testing of other sample types collected contemporaneously from the same patient (2) quantitative-PCR, imaging, GeneXpert (3) clinical adjudication by infectious disease specialists. The performance of mNGS and culture in diagnosis of infectious disease is shown in Table 3 . The sensitivity and specificity of mNGS (all samples) for diagnosing infectious diseases were $79.49 \%$ (95\% confidence interval (Cl) $75.20-83.20 \%)$ and $82.20 \%(95 \% \mathrm{Cl}$ 74.32-88.06\%), respectively. As for conventional test, the sensitivity and specificity of diagnosing infectious disease were $21.25 \%$ (95\% $\mathrm{Cl} 17.25-25.87 \%)$ and $92.86 \%$ (95\% Cl 75.04-98.75\%), respectively. As expected, mNGS increased the sensitivity rate by approximately $60 \%$ in comparison with that of culture (79.49\% vs $21.35 \%$; $P<0.01)$, while the specificity difference was not significant $(83.20 \%$ vs $88.06 \%$; $P>0.05)$. Excluding blood, the sensitivity of mNGS testing were comparable overall among different sample types. ROC analysis of all samples mNGS for the diagnosis of infectious disease yielded an AUC of 0.8823 (95\% Cl, 0.8453-0.9192) (Fig. 3b). Additionally, ROC analysis of blood-mNGS and CSF-mNGS for the diagnosis of infectious disease yielded an AUC of $0.8116(95 \% \mathrm{Cl}, 0.7456-0.8757)$ and $0.7882(95 \% \mathrm{Cl}, 0.6713-0.9051)$, respectively. The highest accuracy of detection from sputum (AUC $=0.9630$ $(95 \% \mathrm{Cl}, 0.7776-1.1428)$ ) and broncho alveolar lavage fluid ( $\mathrm{AUC}=0.9586(95 \% \mathrm{Cl}, 0.8917-1.0256))$. When the threshold was greater than 8.500 , the sensitivity and specificity of mNGS (all samples) were $79.49 \%$ and $82.20 \%$, respectively. The sensitivity and specificity of mNGS testing for bacterial detection based on the composite standard were $78.36 \%$ and $95.31 \%$, respectively, for culture ( $n=397$ samples) compared to $18.03 \%$ and $98.06 \%$, respectively (Fig. 3a). For fungal pathogen detection, the sensitivity and specificity of mNGS were $73.83 \%$ and $94.00 \%$, respectively, for culture ( $n=397$ samples) compared to $34.78 \%$ and $100 \%$, respectively. For Others pathogen detection, including MTB, NTM and atypical pathogens (mycoplasma, chlamydia, rickettsia, spirochete), the sensitivity and specificity of mNGS were $72.22 \%$ and $97.67 \%$, respectively. As for virus pathogen detection, 22 viruses were detected, Epstein Barr virus $(n=61)$ was the most frequently detected virus, followed by Herpesviridae $(n=55)$ and Cytomegalovirus $(n=44)$. The majority of detected viruses were likely concurrent infection unassociated with the clinical presentation. Due to lack of conventional orthogonal testing for viruses and no unified standard for judging whether a virus is pathogenic or virus-carrying, viruses were not separately included in the diagnostic performance assessment.

\section{Comparison of relative pathogen reads of mNGS testing from other samples versus blood}

Sixteen patients in our study had paired other samples and blood samples available for comparative mNGS testing. The number of reads standardized stringently mapped read number at the species level (SDSMRN) was a median 195-fold higher (IQR 33-10748) in the paired other samples than in blood

Page $4 / 14$ 
from the same patient (Fig. 3c). For the same pathogen, fifteen paired other sample (sputum, bronchoalveolar lavage fluid, cerebrospinal fluid and urine) mNGS detected more sequences than blood mNGS in fifteen patients (Fig. 3d). Another patient's blood mNGS identified more Pseudomonas aeruginosa sequences then peritoneal mNGS. Notably, of those 15 patients, there was one patient's sputum mNGS identified more Aspergillus flavus sequences then blood mNGS, but blood mNGS identified E. coli which not identify by sputum mNGS, then E. coli confirmed by culture.

\section{Clinical Impact of mNGS Results on Diagnosis and Treatment}

Consider the possibility of repeated mNGS testing in the same patient for different period or reasons including patient monitoring or suspected falsenegative results from the first mNGS test, the clinical impact was evaluated based on the first mNGS test per patient. A total of 518 mNGS tests were evaluated, mNGS positivity rate was $436 / 518$ (84.17\%). When examining the impact of mNGS results on patient diagnosis and treatment, mNGS results showed a positive or no impact in 267 (51.54\%) and 192 (37.07\%) cases, respectively, whereas a negative impact was observed in 4 cases (0.77\%), which lead to unnecessary antibiotic treatment (Table 4). Positive clinical impact was categorized as empirical treatment continued $(n=105)$, antibiotic escalated $(n=79)$, antibiotic adjust and avoid unnecessary antibiotic treatment (antibiotics were terminate or changed to a narrower range, $n=47)$, rule out infection diseases $(n=20)$, earlier diagnosis and initiation of appropriate therapy $(n=16)$. Among the cases without impact $(n=192)$, mNGS results were considered false or insignificant in 122 cases, of which 47 did not find any additional pathogens and 75 due to identification of a new organisms or deemed contaminants but of no significance. The 70 cases had improved before mNGS results is available, and these patients were defined as having no effect. In terms of patient antibiotic management, mNGS results directly led to a change in treatment (142 of 267 positive impact cases). Among the 142 cases whose treatment changed, almost all patients (118/142) were achieved clinical cure or improvement, while the rest were still seriously due to serious comorbidities despite appropriate treatment. Notably, the 16 early diagnoses made by mNGS test included detection of pathogens encompassing Mycobacterium tuberculosis $(n=10)$, Chlamydia psittaci $(n=2)$, Coxiella burnetiid $(n=2)$, Kaposi sarcoma virus $(n=1)$, Mycobacterium shinjukuense $(n=1)$. This highlights the strength of mNGS in certain cases requiring specific targeted treatment.

To evaluate the potential clinical impact of mNGS for diagnosis of infectious diseases, we selectively enrolled 10 patients with clinically suspected infection but negative in culture (Table 5). An infectious clinical diagnosis had been made by microbial culture and orthogonal microbial testing. Of these 10 mNGS tests, 9 had organisms detected while 1 had no organism detected. Impact was positive in 8 tests and negative in 2 . The details of 10 cases are shown in Table 4. These cases included detection of one to four presumptive causative pathogens including bacteria (eg, Pseudomonas aeruginosa, Mycobacterium tuberculosis), atypical bacteria (eg, c. psittaci), fungus (eg, Cryptococcus, Aspergillus, P. jirovecii), and virus (eg, HRV, EB virus), highlighting the advantage of mNGS in diagnosis complexed etiologies of infection in certain cases.

\section{Discussion}

Here we describe the clinical accuracy validation and practical clinical application of metagenomic next-generation sequencing. Key advances in our research include (1) The accuracy of quantitative microbial cfDNA sequencing tests validated that platform's analysis and interpretation of sequencing date. (2) Detect a wide range of sample types include Sputum, Bronchoalveolar Ivge fluid, Cerebrospinal fluid, Blood et al. (3) The mNGS tests were an inhouse microbiology laboratory, which increases the accuracy of results because of preserves the vitality of the microbes due to reduced turnaround time from bedside to bench. (4) All samples combined both RNA and DNA sequence, most of other studies using DNA sequence only. This approach allowed for simultaneous host transcriptional profiling, enriched for actively transcribing microbes (versus nonviable taxa or latent)(17).

In this study, we systematically compared mNGS identification and culture in a pairwise manner. The founding that mNGS detection rate was significantly higher than culture is in contrast to that recently reported in California where mNGS are not more sensitive than culture when detected Klebsiella pneumoniae(17). The differences between studies may have been driven by discrepancy in the overall level of microbiology laboratory service. Results from different laboratories may not be universally applicable. In addition, culture often reported a single microorganism or negative, which may be due to the competition between the different species of microorganisms or to the administration empirical antibiotics to patients prior to obtaining samples(18). However, mNGS is less affected by prior antibiotic exposure because it does not depend on pathogen survival(19, 20), and for most BALF and sputum samples, mNGS reports up to $5+$ microorganisms. The high detection rate in the present study was closer to that reported in a retrospective study where mNGS-positive samples was significantly higher than that of culture-positive samples(19).

Not surprisingly, mNGS exhibited better diagnostic performance than conventional microbiological culture for detecting bacteria and fungus. The mNGS test was able to detect a possible pathogen in the vast majority of cases. This was consistent with previous research. But among these "mNGS false-negative" cases, mNGS testing failed to detect fifteen cases of M. tuberculosis, a higher number than other false-negative bacteria. This may be due to the difficulty of breaking M. tuberculosis during nucleic acid extraction. Although bead tapping can increase the detection rate of certain bacteria and fungi that contain rigid cell walls, it can simultaneously decrease detection sensitivity by increasing host background release of human DNA(7). Excluding blood samples, the sensitivity of mNGS testing were highly in other samples (ranging from 88.89 to $93.79 \%$ ), which may be due to the low pathogen burden in blood compared with other types of specimens. We showed here that, there was a detected 195-fold higher microbial reads in the paired other samples than in blood from the same patient. Higher level of pathogen load in samples can increase the credibility of mNGS results. The mNGS of sputum and bronchoalveolar lavage fluid outperformed that for other types of samples in the sensitivity of detection of pathogens, the number of reads, the ratio of microbial reads and the rate of genome coverage.

However, multiple probable pathogens were identified in many cases whose clinical symptoms were not concordance with pathogens. The additional detections complicate the interpretation of microbiological reports. Our accuracy validation tests ruled out contamination as the source of these reads, we speculate that the additional pathogens detected were primarily components of the human microbiome and possibly derived from mucosal barrier, cfDNA residue from previous infections and transient bacteremia caused by colonizing bacteria. Dead microorganisms don't cause disease, but they may remain to 
secret detectable small nucleic acid fragments. Therefore, it is recommended that "probable" pathogens should be comprehensively judged by professionals who have certain bioinformatics knowledge and are engaged in clinical infection or clinical microbiology, combined with the reading length of the detected pathogenic microorganisms as well as the differences in the sample types, the phylogeny and underlying molecular biology of the pathogens, the clinical background of patients, imaging data and other laboratory examination results. Considering antimycobacterial, antifungal and antiviral therapies can be toxic and costly, clinicians must be comprehensively judged before initiating antimicrobials therapy for pathogens with few sequences detected, such as M. tuberculosis, Aspergillus and Nocardia. Without correct interpretation, blind use of antimicrobials based on mNGS results will inevitably lead to unnecessarily broaden or prolong antibiotic therapy.

The finding that 84 of 200 (42.0\%) blood mNGS results, 89 of 154 (57.8\%) bronchoalveolar lavage fluid mNGS results lead to positive impact, which indicated alveolar lavage fluid mNGS had a higher positive impact than blood mNGS. Our result is different from recently published in a multicenter in USA where 6/82 (7.3\%) plasma mNGS results were considered positive impact(9). In our study, clinical impact based on the decision made by the treatment team after interpretating the mNGS results and whether leading to a favorable clinical outcome. Another retrospective study on pediatric patients was performed at USA showed mNGS added little diagnostic value when conducted simultaneously with conventional testing, there was no change in management when additional organisms were identified by mNGS in the majority of cases(21), but this study was only assessed the use of mNGS for pediatric patients. In short, the reason why we are inconsistent with the above studies may be due to differences in sample types, patient populations, testing indication and the proportion of critically ill patients. In the past, mNGS has been used as the last test resort for critically ill patients due to price as high as 500 US dollars(22). However, for critically ill patients, the clinical outcome may be poor, although mNGS results may be useful in guiding antibiotic management. In our study, we tend to order mNGS as a first-line test for critically ill patients and patients with suspected atypical pathogen infection. This may explain why this study has higher positive clinical impact versus previous study. Nevertheless, 16 of 518 (3.1\%) cases included condition were very critically and clinical impact was difficult to ascertain.

The limitations of this study include the following. First, the number of some sample types like sputum and peritoneal is low. Second, this study is a singlecentral study with limited representativeness, which conclusion cannot be generalized to a broader scale of laboratory. Third, some patients were given empirical antimicrobic treatment before sampling, we did not conduct a longitudinal assessment of the effects of antimicrobial administration before and after on mNGS and culture results.

In summary, here we present a retrospective study to fully evaluate the utility of mNGS for the diagnosis of suspected infectious diseases and the real-world clinical impact. More data are needed to determine the clinical impact of mNGS and to instruct which types of samples and patient populations are more favorable for mNGS testing. Nonetheless, this study suggests that mNGS testing may be practical in these scenarios: (1) as first-line detection tool for critically ill patients and immunosuppression patients who need to identify pathogens as soon as possible, (2) for identification of unknown, rare and atypical pathogens. (3) as a complementary test for culture-negative pathogens.

\section{Conclusion}

mNGS significantly improved sensitivity for bacteria and fungi identification, is a valuable tool for for identifying infectious patients and could optimize antimicrobial therapy to improve the clinical outcomes. Multicenter and prospective studies with larger samples will be necessary to validate the clinical efficacy and diagnostic value of mNGS in the future.

\section{Abbreviations}

mNGS: Metagenomics next-generation sequencing; SDSMRN: standardized stringently mapped read number at the species level; BAL: Bronchoalveolar lavage fluid; CSF: cerebrospinal fluid; MTB: Mycobacterium tuberculosis; NTB: Non-tuberculous mycobacteria.

\section{Declarations}

\section{Acknowledgments}

The authors thank all the staff members at our institution.

\section{Authors' contributions}

ZR conceived, designed, and supervised the study. XYH acquired and interpreted the data. BYX, YL, LY and WHW conducted the clinical work associated with the study. XDR provided the technical support. XDR and ZW verified the underlying data. XYH wrote the draft, and ZW, XDR, ZR revised it. All authors read and approved the final version of the manuscript. The corresponding author attests that all listed authors meet the authorship criteria and that no others meeting the criteria have been omitted.

\section{Funding}

This study was supported by the National Natural Science Foundation of Jiangxi Province, China (Grant No. 20192BAB205088), the Natural Science Foundation of Jiangxi Province Department of Education, China (Grant No.GJJ180120) and the National Natural Science Foundation of China (Grant 
No.61861028); the Young Scientists Fund of Jiangxi Province Department of Science and Technology, China (Grant No.20171BAB212010); and the Chinese Medicine Scientific Research Program (key) of Jiangxi Province Department of Health Commission, China (Grant No.2017Z015) from conception to publication.

\section{Availability of data and materials}

The original contributions presented in the study are included in the article/Supplementary Material; further inquiries can be directed to the corresponding author. Full sequencing data is unavailable due to patient confidentiality.

\section{Ethics approval and consent to participate}

Ethical approval was approved by the ethical committee of the First Affiliated Hospital of Nanchang University (Jiangxi Province, China). Informed consents were signed by patients or surrogates.

\section{Consent for publication}

Written informed consent was obtained from the patient for publication of this case report and any accompanying images. A copy of the written consent is available for review by the Editor of this journal.

\section{Competing interests}

The authors declare that there is no competing interest.

\section{References}

1. Lozano R, Naghavi M, Foreman K, Lim S, Shibuya K, Aboyans V, et al. Global and regional mortality from 235 causes of death for 20 age groups in 1990 and 2010: a systematic analysis for the Global Burden of Disease Study 2010. Lancet. 2012;380(9859):2095-128.

2. Sankar J, Garg M, Ghimire JJ, Sankar MJ, Lodha R, Kabra SK. Delayed Administration of Antibiotics Beyond the First Hour of Recognition Is Associated with Increased Mortality Rates in Children with Sepsis/Severe Sepsis and Septic Shock. J Pediatr. 2021;233:183-90 e3.

3. Rea B, Maisel JR, Glaser L, Alby K. Identification of Clinically Relevant Mycobacterial Species After Extended Incubation Times in the BACTEC MGIT System. Am J Clin Pathol. 2019;151(1):63-7.

4. Huang H, Deng J, Qin C, Zhou J, Duan M. Disseminated Coinfection by and in a Non-HIV Case. Infect Drug Resist. 2021;14:3619-25.

5. Peri AM, Harris PNA, Paterson DL. Culture-independent detection systems for bloodstream infection. Clin Microbiol Infect. 2021.

6. Zhang H, Han Y, Jin Z, Ying Y, Lan F, Huang H, et al. Detection of Viruses by Multiplex Real-Time Polymerase Chain Reaction in Bronchoalveolar Lavage Fluid of Patients with Nonresponding Community-Acquired Pneumonia. Can Respir J. 2020;2020:8715756.

7. Gu W, Deng X, Lee M, Sucu YD, Arevalo S, Stryke D, et al. Rapid pathogen detection by metagenomic next-generation sequencing of infected body fluids. Nat Med. 2021;27(1):115-24.

8. Chiu CY, Miller SA. Clinical metagenomics. Nat Rev Genet. 2019;20(6):341-55.

9. Hogan CA, Yang S, Garner OB, Green DA, Gomez CA, Dien Bard J, et al. Clinical Impact of Metagenomic Next-Generation Sequencing of Plasma Cell-Free DNA for the Diagnosis of Infectious Diseases: A Multicenter Retrospective Cohort Study. Clin Infect Dis. 2021;72(2):239-45.

10. Cao J, Cai Q, Su W, Ge Z, Zhao H, Zhou X, et al. Case Report: Metagenomic Next-Generation Sequencing Confirmed a Case of Central Nervous System Infection With in Non-endemic Areas. Front Med (Lausanne). 2021;8:723197.

11. Shi L, Xia H, Moore MD, Deng C, Li N, Ren H, et al. Metagenomic Next-Generation Sequencing in the Diagnosis of HHV-1 Reactivation in a Critically III COVID-19 Patient: A Case Report. Front Med (Lausanne). 2021;8:715519.

12. Chen Y, Feng W, Ye K, Guo L, Xia H, Guan Y, et al. Application of Metagenomic Next-Generation Sequencing in the Diagnosis of Pulmonary Infectious Pathogens From Bronchoalveolar Lavage Samples. Front Cell Infect Microbiol. 2021;11:541092.

13. Yan G, Liu J, Chen W, Chen Y, Cheng Y, Tao J, et al. Metagenomic Next-Generation Sequencing of Bloodstream Microbial Cell-Free Nucleic Acid in Children With Suspected Sepsis in Pediatric Intensive Care Unit. Front Cell Infect Microbiol. 2021;11:665226.

14. Sun T, Wu X, Cai Y, Zhai T, Huang L, Zhang Y, et al. Metagenomic Next-Generation Sequencing for Pathogenic Diagnosis and Antibiotic Management of Severe Community-Acquired Pneumonia in Immunocompromised Adults. Front Cell Infect Microbiol. 2021;11:661589.

15. Zhou H, Larkin PMK, Zhao D, Ma Q, Yao Y, Wu X, et al. Clinical Impact of Metagenomic Next-Generation Sequencing of Bronchoalveolar Lavage in the Diagnosis and Management of Pneumonia: A Multicenter Prospective Observational Study. J Mol Diagn. 2021;23(10):1259-68.

16. Blauwkamp TA, Thair S, Rosen MJ, Blair L, Lindner MS, Vilfan ID, et al. Analytical and clinical validation of a microbial cell-free DNA sequencing test for infectious disease. Nat Microbiol. 2019;4(4):663-74. 
17. Langelier C, Kalantar KL, Moazed F, Wilson MR, Crawford ED, Deiss T, et al. Integrating host response and unbiased microbe detection for lower respiratory tract infection diagnosis in critically ill adults. Proc Natl Acad Sci U S A. 2018;115(52):E12353-E62.

18. Wang J, Zeng Y, Wang S, Liu H, Zhang D, Zhang W, et al. Swine-Derived Probiotic Lactobacillus plantarum Inhibits Growth and Adhesion of Enterotoxigenic Escherichia coli and Mediates Host Defense. Front Microbiol. 2018;9:1364.

19. Miao Q, Ma Y, Wang Q, Pan J, Zhang Y, Jin W, et al. Microbiological Diagnostic Performance of Metagenomic Next-generation Sequencing When Applied to Clinical Practice. Clinical Infectious Diseases. 2018;67(suppl_2):S231-S40.

20. Gosiewski T, Ludwig-Galezowska AH, Huminska K, Sroka-Oleksiak A, Radkowski P, Salamon D, et al. Comprehensive detection and identification of bacterial DNA in the blood of patients with sepsis and healthy volunteers using next-generation sequencing method - the observation of DNAemia. Eur $\mathrm{J}$ Clin Microbiol Infect Dis. 2017;36(2):329-36.

21. Niles DT, Wijetunge DSS, Palazzi DL, Singh IR, Revell PA. Plasma Metagenomic Next-Generation Sequencing Assay for Identifying Pathogens: a Retrospective Review of Test Utilization in a Large Children's Hospital. J Clin Microbiol. 2020;58(11).

22. Wilson MR, Sample HA, Zorn KC, Arevalo S, Yu G, Neuhaus J, et al. Clinical Metagenomic Sequencing for Diagnosis of Meningitis and Encephalitis. N Engl J Med. 2019;380(24):2327-40.

\section{Tables}

Table 1 Accuracy validation for the mNGS assay

\begin{tabular}{|c|c|c|c|c|c|c|c|}
\hline Pathogen & & D1 (Reads) & D2 (Reads) & D3 (Reads) & D4 (Reads) & D5 (Reads) & Did mNGS detected this pathogen? \\
\hline \multirow[t]{2}{*}{ Listeria grayi } & Genus & 12182 & 1633 & 86 & 932 & 2179 & \multirow[t]{2}{*}{ Y } \\
\hline & Species & 12173 & 1632 & 86 & 929 & 2179 & \\
\hline \multirow[t]{2}{*}{ Haemophilus parainfluenzae } & Genus & 14289 & 1875 & 102 & 909 & 2482 & \multirow[t]{2}{*}{ Y } \\
\hline & Species & 13394 & 1765 & 102 & 909 & 2482 & \\
\hline \multirow[t]{2}{*}{ Acinetobacter junii } & Genus & 12724 & 1772 & 162 & 2885 & 11109 & \multirow[t]{2}{*}{$\mathrm{Y}$} \\
\hline & Species & 11614 & 1593 & 110 & 1294 & 3192 & \\
\hline \multirow[t]{2}{*}{ Rhodococcus equi } & Genus & 3505 & 435 & 22 & 135 & 260 & \multirow[t]{2}{*}{$\mathrm{Y}$} \\
\hline & Species & 3483 & 432 & 22 & 135 & 260 & \\
\hline \multirow[t]{2}{*}{ Micrococcus luteus } & Genus & 4389 & 584 & 36 & 367 & 1549 & \multirow[t]{2}{*}{ Y } \\
\hline & Species & 4340 & 577 & 36 & 359 & 1126 & \\
\hline \multirow[t]{2}{*}{ Neisseria sicca } & Genus & 6317 & 910 & 80 & 370 & 1176 & \multirow[t]{2}{*}{$\mathrm{Y}$} \\
\hline & Species & 5349 & 724 & 57 & 294 & 885 & \\
\hline \multirow[t]{2}{*}{ Pseudomonas fluorescens } & Genus & 46383 & 6055 & 1535 & 20586 & 81858 & \multirow[t]{2}{*}{$\mathrm{Y}$} \\
\hline & Species & 43931 & 5328 & 557 & 3224 & 12020 & \\
\hline \multirow[t]{2}{*}{ Aeromonoas hydrophila } & Genus & 14814 & 1623 & 217 & 887 & 3234 & \multirow[t]{2}{*}{ Y } \\
\hline & Species & 13911 & 1582 & 169 & 818 & 3030 & \\
\hline \multirow[t]{2}{*}{ Legionella pneumophila } & Genus & 31243 & 4065 & 371 & 2185 & 7911 & \multirow[t]{2}{*}{$\mathrm{Y}$} \\
\hline & Species & 31243 & 4009 & 363 & 2098 & 7594 & \\
\hline \multirow[t]{2}{*}{ Candida lusitaniae } & Genus & 3061 & 304 & 26 & 221 & 518 & \multirow[t]{2}{*}{ Y } \\
\hline & Species & 2790 & 304 & 26 & 219 & 518 & \\
\hline
\end{tabular}


Table 2 Patient and sample characteristics

\begin{tabular}{|c|c|}
\hline Characteristics & Value \\
\hline \multicolumn{2}{|l|}{ Patient demographics $(n=518)$} \\
\hline \multicolumn{2}{|l|}{ Gender, $n(\%)$} \\
\hline Female & $341(65.83)$ \\
\hline Male & $177(34.17)$ \\
\hline \multicolumn{2}{|l|}{ Age (years) } \\
\hline Median (IQR) & $56(43-67)$ \\
\hline Range & $1-96$ \\
\hline \multicolumn{2}{|l|}{ Hospitalization n (\%) } \\
\hline In hospital & $518(100.0)$ \\
\hline In intensive care unit & $139(26.83)$ \\
\hline \multicolumn{2}{|l|}{ Primary clinical symptoms n (\%) } \\
\hline Fever & $245(47.30)$ \\
\hline Cough & $107(20.66)$ \\
\hline Increased sputum production & $74(14.29)$ \\
\hline Fatigue & $58(11.20)$ \\
\hline Chest congestion & $57(11.00)$ \\
\hline Dizziness & $52(10.04)$ \\
\hline headache & $50(9.65)$ \\
\hline Shortness of breath & $40(7.72)$ \\
\hline \multicolumn{2}{|l|}{ Basic illness n (\%) } \\
\hline Hypertension & $125(24.13)$ \\
\hline Diabetes & $53(10.23)$ \\
\hline Chronic liver diseases & $51(9.85)$ \\
\hline Chronic lung diseases & $30(5.79)$ \\
\hline Cerebrovascular diseases & $24(4.63)$ \\
\hline Cardiovascular Diseases & $13(2.51)$ \\
\hline Chronic kidney diseases & $13(2.51)$ \\
\hline Organ transplantation & $6(1.16)$ \\
\hline Autoimmunity disease & $5(0.97)$ \\
\hline
\end{tabular}


Table 3 Diagnostic performance of Pathogen for mNGS and traditional methods compared with the clinical final adjudication Sensitivity $(95 \% \mathrm{Cl}) \quad$ Specificity $(95 \% \mathrm{Cl})$

\begin{tabular}{|c|c|c|}
\hline \multicolumn{3}{|c|}{ All samples } \\
\hline mNGS & $79.49 \%(75.20-83.20 \%)$ * & $82.20 \%(74.32-88.06 \%)$ \\
\hline culture & $21.25 \%(17.25-25.87 \%)$ & $92.86 \%$ (75.04-98.75\%) \\
\hline AMT & $41.42 \%(36.36-46.65 \%)$ & $96.43 \%$ (79.76-99.81\%) \\
\hline \multicolumn{3}{|l|}{ Blood } \\
\hline mNGS & $67.44 \%(58.95-74.92 \%)$ * & $82.81 \%(71.79-90.12 \%)$ \\
\hline culture & 7.03\% (3.47\%-13.31\%) & $100.0 \%(69.87 \%-100.0 \%)$ \\
\hline AMT & $32.81 \%(24.93-41.75 \%)$ & $100.0 \%(69.87 \%-100.0 \%)$ \\
\hline \multicolumn{3}{|l|}{ BALF } \\
\hline mNGS & $93.79 \%(88.63-96.70 \%)$ * & $87.50 \%(52.91-99.36 \%)$ \\
\hline culture & $36.29 \%(27.99-45.46 \%)$ & $50.00 \%(2.67-97.33 \%)$ \\
\hline AMT & $57.25 \%(48.06-65.99 \%)$ & $50.00 \%(2.67-97.33 \%)$ \\
\hline \multicolumn{3}{|l|}{ CSF } \\
\hline mNGS & $88.89 \%(74.69-95.59 \%)$ * & $62.50 \%(42.71-78.84 \%)$ \\
\hline culture & $6.52 \%(1.70-18.93 \%)$ & $100.0 \%(56.09-100.0 \%)$ \\
\hline AMT & $17.39 \%(8.32-31.95 \%)$ & $100.0 \%(56.09-100.0 \%)$ \\
\hline \multicolumn{3}{|l|}{ Sputum } \\
\hline mNGS & $92.59 \%(76.63-98.68 \%)$ * & $100.0 \%(17.77-100.0 \%)$ \\
\hline culture & $64.00 \%(42.62-81.29 \%)$ & $100.0 \%(5.46-100.0 \%)$ \\
\hline AMT & $68.00 \%(46.44-84.27 \%)$ & $100.0 \%(5.46-100.0 \%)$ \\
\hline
\end{tabular}

annotation: AMT: all microbiological tests

* The sensitivity of mNGS reached statistical significance compared to culture (McNemar-test $P<0.001)$

Table 4. Clinical Impact and Role of mNGS Result

Clinical impact Treatment changes based on mNGS

Positive impact $n=267 ; 51.54 \% \quad$ Empirical treatment continued $(n=105 ; 20.27 \%)$

Antibiotic escalated ( $n=79 ; 15.25 \%)$

Antibiotic adjust and avoid unnecessary antibiotic treatment $(n=47 ; 9.07 \%)$

Ruled out active infection ( $n=20 ; 3.86 \%)$

Earlier diagnosis and initiation of appropriate therapy $(n=16 ; 3.09 \%)$

Negative impact $(n=4 ; 0.77 \%)$

Unnecessary antibiotic treatment $(n=4 ; 0.77 \%)$

No impact $(n=192 ; 37.07 \%)$

Results considered false or insignificant $(n=122 ; 23.55 \%)$

Patient has improved before result is available $(n=70 ; 13.51 \%)$

Indeterminate $(n=55 ; 10.62 \%)$

Earlier discharge $(n=27 ; 5.21 \%)$

Patient's condition is critically $(16 ; 3.09 \%)$

Patient's information is incomplete $(n=12 ; 2.32 \%)$ 
Table 5 Case series of mNGS testing in patients with probable infection but negative in culture

\begin{tabular}{|c|c|c|c|c|c|c|c|}
\hline $\begin{array}{l}\text { case } \\
\text { ID }\end{array}$ & $\begin{array}{l}\text { Sample } \\
\text { type }\end{array}$ & presentation & mNGS result & $\begin{array}{l}\text { culture } \\
\text { result }\end{array}$ & Clinical diagnosis & $\begin{array}{l}\text { Clinical } \\
\text { impact }\end{array}$ & Detail \\
\hline S28 & BAL & $\begin{array}{l}\text { cough and } \\
\text { expectoration }\end{array}$ & Human rhinovirus $\mathrm{A} 28$ & negative & viral pneumonia & Positive & $\begin{array}{l}\text { mNGS result prompted } \\
\text { anti-viral therapy with } \\
\text { ribavirin. }\end{array}$ \\
\hline S40 & BAL & pulmonary shadow & cryptococcus neoformans & negative & $\begin{array}{l}\text { pulmonary } \\
\text { cryptococcosis }\end{array}$ & Positive & $\begin{array}{l}\text { mNGS diagnosed } \\
\text { pulmonary cryptococcosis, } \\
\text { which enabled targeted } \\
\text { antifungal therapy. }\end{array}$ \\
\hline S56 & BAL & fever & chlamydia psittaci & negative & pneumonia (c. psittaci ) & Positive & $\begin{array}{l}\text { mNGS result prompted } \\
\text { early definite diagnosis } \\
\text { and targeted therapy with } \\
\text { doxycycline }\end{array}$ \\
\hline S62 & BAL & back pain & $\begin{array}{l}\text { Pseudomonas aeruginosa, } \\
\text { candida peridosmooth, } \\
\text { pneumocystis jirovecii, } \\
\text { Epstein-Barr virus }\end{array}$ & negative & $\begin{array}{l}\text { Bacterial, fungal, viral } \\
\text { pneumonia }\end{array}$ & Positive & $\begin{array}{l}\text { mNGS identified mixed } \\
\text { infections, which allowed } \\
\text { narrowing of targeted } \\
\text { therapy with ganciclovir } \\
\text { and Sulfamethoxazole }\end{array}$ \\
\hline S218 & Blood & fever & $\begin{array}{l}\text { Klebsiella pneumoniae, } \\
\text { parvovirus }\end{array}$ & negative & $\begin{array}{l}\text { Septic shock (K. } \\
\text { pneumoniae) }\end{array}$ & Positive & $\begin{array}{l}\text { mNGS not detected Gram- } \\
\text { positive bacteria. This } \\
\text { prompted discontinuation } \\
\text { of empiric linezolid. }\end{array}$ \\
\hline S387 & CSF & Recurrent fever & Mycobacterium tuberculosis & negative & $\begin{array}{l}\text { Tubercular meningitis } \\
\text { (M. tuberculosis) }\end{array}$ & Positive & $\begin{array}{l}\text { mNGS result prompted } \\
\text { early definite diagnosis } \\
\text { and initiate } \\
\text { targetedantituberculous } \\
\text { therapy }\end{array}$ \\
\hline S427 & Marrow & fever, chills & negative & negative & Autoimmune disease & Positive & $\begin{array}{l}\text { mNGS result prompted } \\
\text { clinician ruled out } \\
\text { infection diseases and } \\
\text { initiate empirical } \\
\text { immunosuppression }\end{array}$ \\
\hline S514 & Sputum & $\begin{array}{l}\text { osphyalgia, } \\
\text { kidney stones }\end{array}$ & Aspergillus flavus, EB virus & negative & $\begin{array}{l}\text { Fungal septicemia, E. } \\
\text { coli sepsis }\end{array}$ & Positive & $\begin{array}{l}\text { mNGS result was used to } \\
\text { initiate antifungal therapy } \\
\text { with voriconazole } 2 \text { days } \\
\text { earlier than culture }\end{array}$ \\
\hline S37 & BAL & $\begin{array}{l}\text { cough, chest } \\
\text { congestion }\end{array}$ & $\begin{array}{l}\text { Rothia mucilaginos, } \\
\text { Pseudomonas aeruginosa, } \\
\text { Streptococcus australis }\end{array}$ & negative & $\begin{array}{l}\text { P.aeruginosa } \\
\text { pneumonia, Fungal } \\
\text { pneumonia }\end{array}$ & negative & $\begin{array}{l}\text { mNGS result led to } \\
\text { additional anti-Gram- } \\
\text { positive bacterial therapy } \\
\text { with linezolid }\end{array}$ \\
\hline S314 & Blood & fever & Human mastadenovirus B & negative & Autoimmune disease & negative & $\begin{array}{l}\text { mNGS result led to } \\
\text { additional anti-viral } \\
\text { therapy with ribavirin }\end{array}$ \\
\hline
\end{tabular}

Figures 

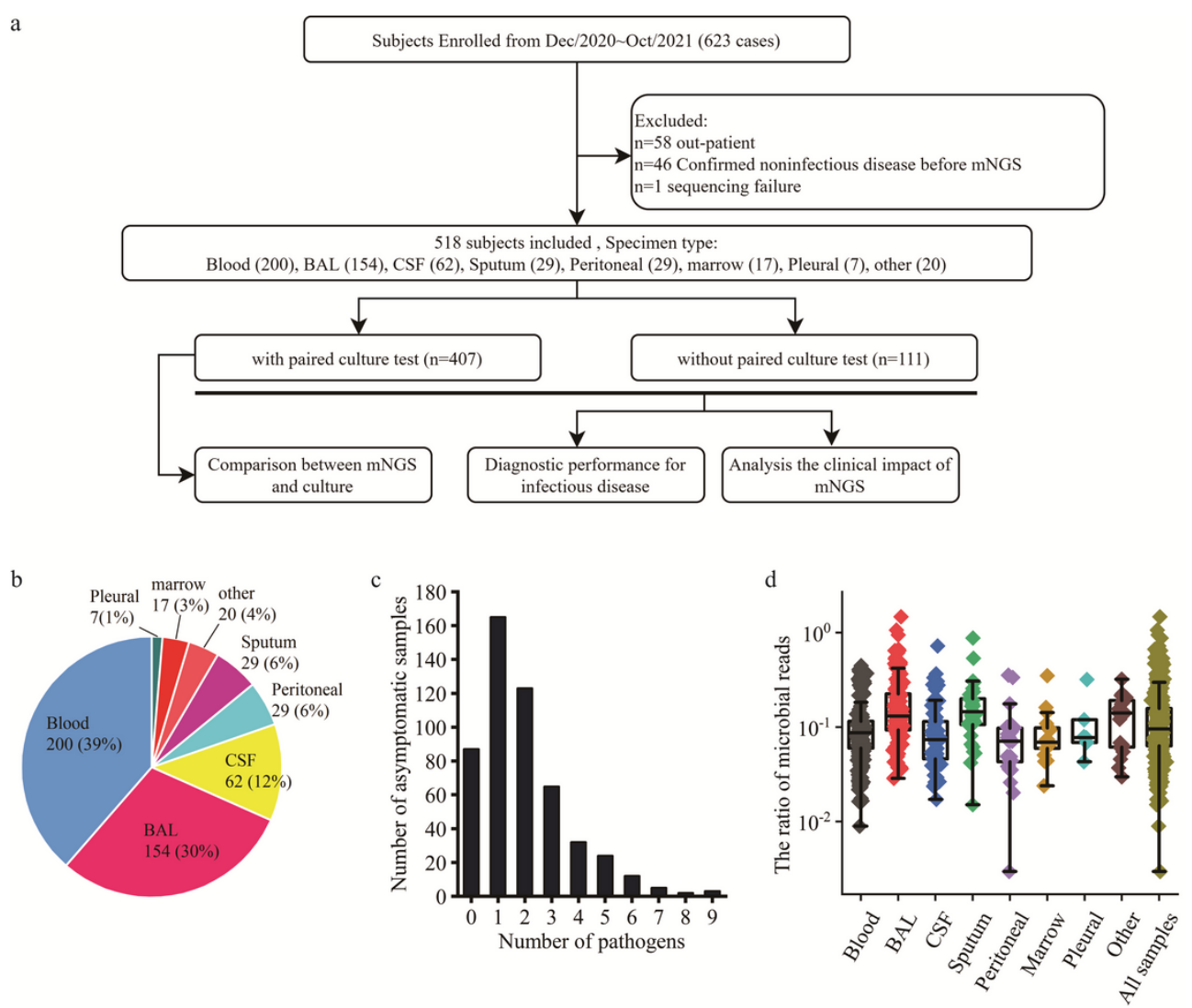

e

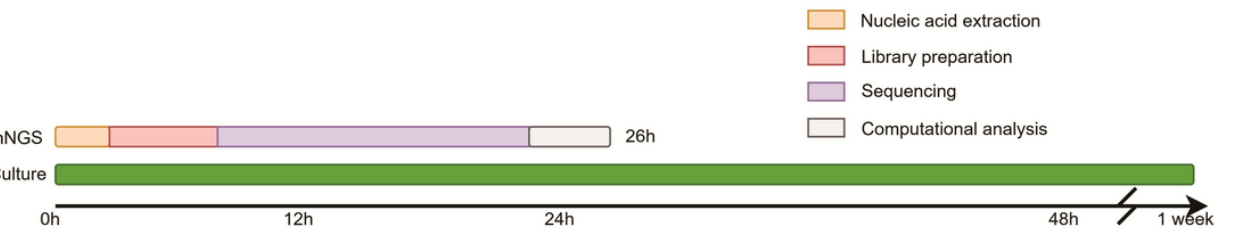

\section{Figure 1}

Study workflow and sample characteristics

a. Flowchart of subject selection, sample classification, and comparison. From 626 samples, a total of 518 were selected for further analysis. Samples were divided into with paired culture test and without paired culture test. Samples with paired culture test were used for the comparison analysis of metagenomic next-generation sequencing (mNGS) and culture, while all patients were used to evaluate the diagnostic performance and the clinical impact of mNGS. b. The pie chart demonstrates the sample types analyzed in the study. c. The frequency of microbial detection in 518 samples. $d$. The ratio of microbial reads is calculated using microbial readings divided by total sequencing readings, stratified by sample type. The ratio of microbial reads is shown as boxplots (with median and interquartile range). e. Comparison of time consumption between mNGS and CMT for pathogen identification. Culture-based pathogen identification was significantly longer than mNGS test. 


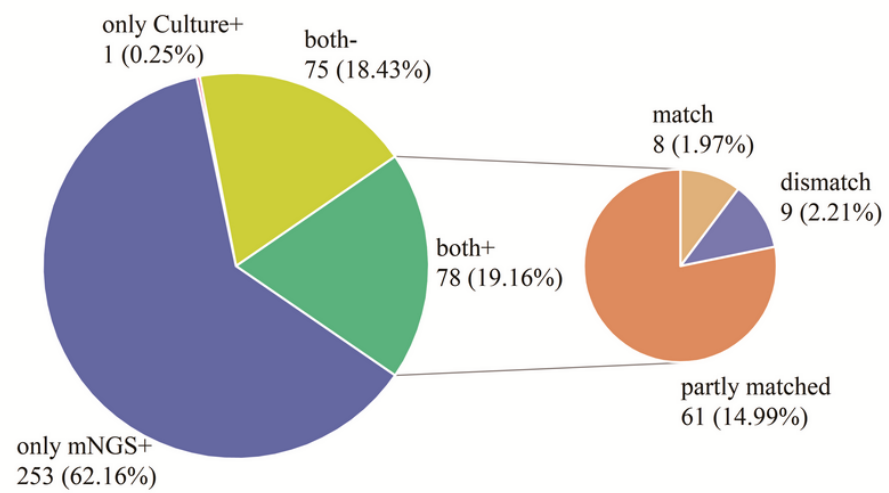

b

Bacteria

Klebsiella pneumoniae

Acinetobacter baumanii

Streptococcus

Escherichia coli

Stenotrophomonas maltophilia

Pseudomonas aeruginosa

Nontuberculosis mycobacteria

Enterococcus

Mycobacterium tuberculosis

Chlamydia psittaci

Staphylococcus aureus

Staphylococcus haemolyticus

Hemophilus parainfluenzae

Corynebacterium striata

Orientia tsutsugamushi

Fungus

Candida

Aspergillus

Pneumocystis pneumonia

Cryptococcus

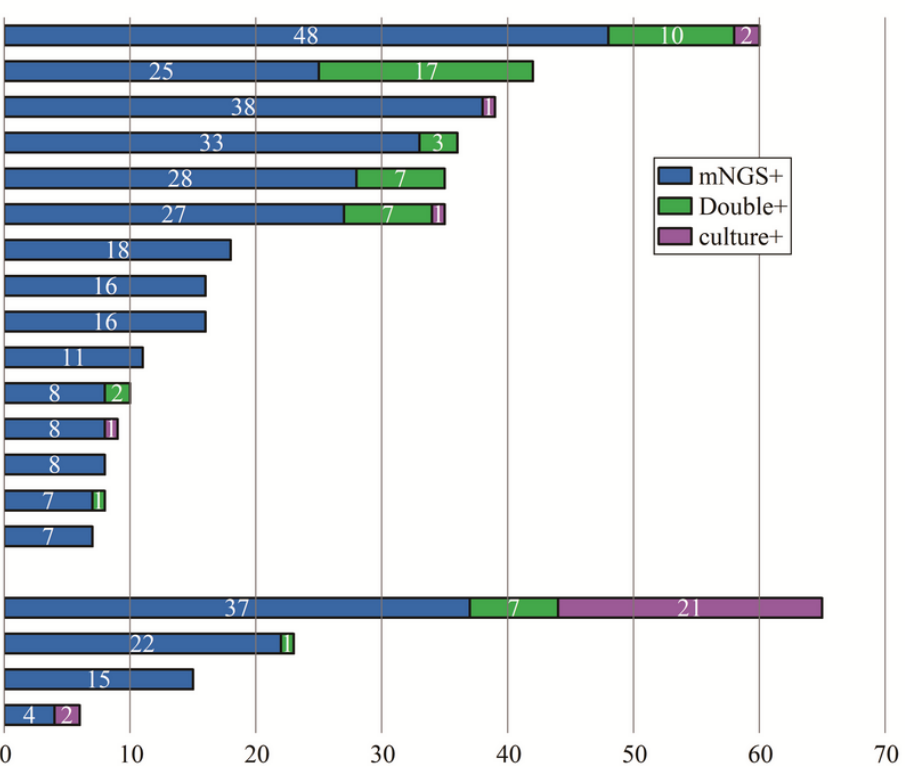

\section{Figure 2}

Comparison of Pathogenic Detection Between mNGS and culture

a. Pie chart demonstrating the positivity distribution of mNGS and culture for samples with paired culture test. For the double-positive subset, a high proportion of partial matching (61/78) (at least 1 pathogen identified in the test was confirmed by the other), with 8 complete matching and 9 conflicts between mNGS and culture results. b. Comparison of culture and mNGS identification in terms of pathogen species. A total of 82 different pathogens were detected in patients with a final diagnosis of infectious disease. The 15 bacteria and 4 fungi with the highest frequency were detected as shown in the figure, and their corresponding frequencies were plotted as histograms. Blue bars indicate microbes detected by mNGS only (mNGS+culture-). Green bars indicate microbes detected by mNGS and predicted to be pathogens by culture (mNGS+culture+). Red bars indicate microbes detected by culture only (mNGS-culture+). 
mNGS: Bacteria(n=502) (with out MTB/NTM) mNGS: Fungi(n=502) mNGS: others $(\mathrm{n}=497) \quad$ culture: Bacteria( $\mathrm{n}=395)$
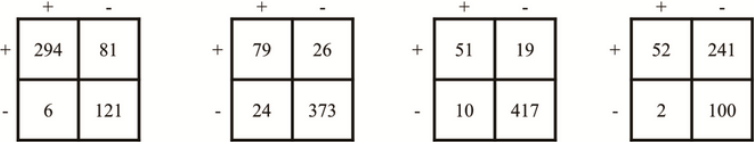

Sens $/$ spec $=78.40 / 95.27 \%$ Sens/spec $=75.23 / 93.95 \%$ Sens $/$ spec $=72.85 / 97.65 \%$ Sens $/$ spec $=17.74 / 98.03 \%$ Sens $/$ spec $=34.78 / 100.0 \%$

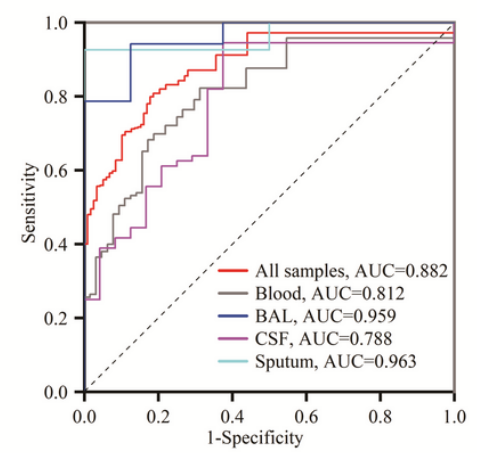

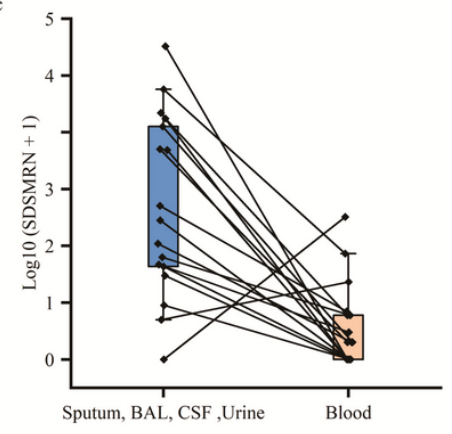

d

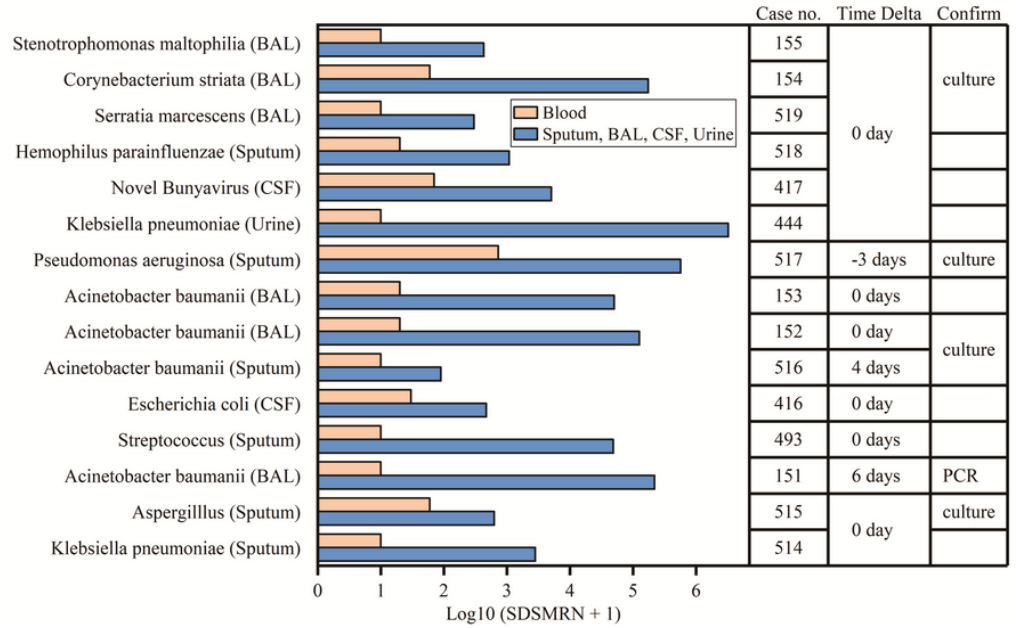

Figure 3

Accuracy of mNGS testing and comparison of relative pathogen reads in different samples

a. Contingency tables formatted in a $2 \times 2$ manner showing the respective diagnostic performance of mNGS and culture testing for bacteria and Fungi. *: Others including MTB, NTM and atypical pathogens (for example, mycoplasma, chlamydia, rickettsia, spirochete) b. ROC curves of mNGS ( $\mathrm{n}=518$ samples) based on a composite standard. c. Comparison of the number of reads in paired other samples and blood sample mNGS for different pathogens. The Log10(SDSMRN + 1) of pathogens detected by paired other samples and blood sample mNGS were shown in blue and orange. d. Bar plot of SDSMRN corresponding to 15 pathogens in paired other samples and blood sample from sixteen patients. The vertical bars represent the number of reads. The first checkbox on the right denotes microorganisms that were confirmed by culture and/or PCR. The second checkbox on the right denote difference in days between paired other samples and blood sample collection; for example, 4 days refers to blood sample collection done 4 days before paired other samples collection. Abbreviations: SDSMRN: standardized stringently mapped read number at the species level.

\section{Supplementary Files}

This is a list of supplementary files associated with this preprint. Click to download.

- SupplementaryTableS1.xlsx

- SupplementaryTables2.xlsx 